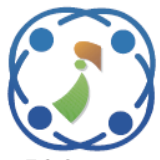

\title{
Mixed Best Members Based Optimizer for Solving Various Optimization Problems
}

\author{
Sajjad Amiri Doumari ${ }^{1}$ \\ Mohammad Dehghani ${ }^{2 *}$ \\ Fatemeh Ahmadi Zeidabadi ${ }^{1}$ \\ Om Parkash Malik ${ }^{3}$ \\ ${ }^{1}$ Department of Mathematics and Computer Sciences, Sirjan University of Technology, Sirjan, Iran \\ ${ }^{2}$ Department of Electrical and Electronics Engineering, Shiraz University of Technology, Shiraz, Iran \\ ${ }^{3}$ Department of Electrical Engineering, University of Calgary, Calgary Alberta, Canada \\ * Corresponding author's Email: adanbax@gmail.com
}

\begin{abstract}
Numerous designed optimization problems in different disciplines of science should be solved using appropriate techniques. population based optimization algorithms are one of the powerful tools in solving optimization problems. The innovation of this paper is to present a new optimization algorithm called Mixed Best Members Based Optimizer (MBMBO) that can be used to solve various optimization problems. The main idea in designing the proposed MBMBO algorithm is to create a mixed member of several top members of the population in order to guide and update the algorithm population. The main feature and advantage of the MBMBO is the lack of control parameters. Therefore, the proposed MBMBO does not need to adjust the parameter. The various steps of the MBMBO are described and then mathematically modeled for implementation in solving optimization problems. The performance of the MBMBO in solving optimization problems is evaluated on a set of twenty-three standard objective functions. These objective functions are of three different types, including seven unimodal objective functions, six high dimensional multi-model objective functions, and ten fixed dimensional multi-model objective functions. The results of evaluation of single-model objective functions indicate the high exploitation power and also the results of evaluation of multi-model objective functions indicate the high exploration power of the proposed MBMBO algorithm. Also, the results obtained from the simulation of the MBMBO are compared with the results of eight other well-known optimization algorithms including Genetic Algorithm (GA), Particle Swarm Optimization (PSO), Gravitational Search Algorithm (GSA), Teaching Learning-Based Optimization (TLBO), Gray Wolf Optimizer (GWO), Emperor Penguin Optimizer (EPO), Hide Objects Game Optimization (HOGO), and Shell Game Optimization (SGO). The results of optimizing the objective functions of unimodal and multi-modal types using MBMBO show the acceptable ability of the proposed algorithm to provide suitable solutions. Comparison of the simulation results shows that the proposed MBMBO is much more competitive than the other eight optimization algorithms.
\end{abstract}

Keywords: Optimization, Optimization algorithm, Optimization problems, Mixed members, Best members.

\section{Introduction}

The optimization problem is simply defined as a problem that has several feasible solutions. As a result, optimization is finding the most appropriate feasible solution for an optimization problem considering all constraints. From a mathematical point of view, each optimization problem consists of three main parts: variables, constraints, and objective functions. After mathematical modeling, the optimization problem must be solved using the appropriate method. Population based optimization algorithms is one of the efficient methods in providing suitable solutions for various optimization problems in different fields of science.

Optimization algorithms are methods that are able to provide appropriate solutions based on random search without the need to derivative and gradient information. Optimization algorithms are designed based on the modeling of various natural phenomena, physical laws, game rules, and other 
processes that can be used to design an optimizer. For example, modelling the behaviour of ants to find the shortest path is used in the design of the Ant Colony Optimization (ACO) algorithm [1]. Hook's law simulation in a system of weights and springs has been used in the design of the Spring Search Algorithm (SSA) [2]. simulation of darts game and player behaviour are used in the design of the Darts Game Optimizer (DGO) algorithm [3]. An optimization algorithm first delivers solutions randomly, then in an iteration-based process, those solutions are improved at each iteration. At the end of the algorithm iterations, the best solution obtained from the optimization algorithm is available. The main difference between optimization algorithms is how to update the population and improve solutions in each iteration.

Each optimization problem has a basic solution called global optimal. The important point is that the solutions presented using optimization algorithms are not necessarily global optimal. For this reason, the solutions obtained from the optimization algorithms are called quasi-optimal. In general, in comparison the performance of optimization algorithms, an algorithm is superior which can provide the best quasi-optimal solution close to the global optimal. For this reason, numerous optimization algorithms have been designed by researchers to provide suitable quasi-optimal solutions. In this regard, optimization algorithms have been applied by scientists in various fields such as energy [4, 5], protection [6], Energy Commitment (EC) [7, 8], placement of electrical equipment [9-11], operation of the electricity network [12, 13], and energy carriers $[14,15]$ to achieve the optimal solution.

The main contribution in this paper is designing a new population based optimization algorithm called Mixed Best Members Based Optimizer (MBMBO) for solving various optimization problems. The main idea in designing the proposed MBMBO is to update the population of the algorithm based on a mixed member made up of several top members of the population. The special advantage of the MBMBO is the effective use of information from population members and thus increase the exploration power of proposed optimizer in achieving quasi-optimal solutions. The main feature of the proposed MBMBO is lack of control parameters and no need to adjust the parameter. The theory of the proposed MBMBO is described and then mathematically is modeled for implementation on optimization problems. The performance of the MBMBO in providing suitable quasi-optimal solutions is tested on a set of twentythree standard objective functions of three different types unimodal, high-dimensional multimodal, and fixed dimensional multimodal functions. Also, the obtained optimization results using the MBMBO are compared with the performance of eight other wellknown optimization algorithms.

The rest of the paper is as follows: Section 2 provides an overview of optimization algorithms. In section 3, the proposed MBMBO is introduced. The simulation studies and the results are presented in Section 4. Finally, conclusions and some suggestions for future studies are provided in Section 5.

\section{Background}

As mentioned, many optimization algorithms inspired by different ideas have been designed to be used in solving various optimization problems in different disciplines of science. Although the idea of designing optimization algorithms is different, all of these methods provide a solution to the optimization problem based on a random search in the problemsolving space. Therefore, the main criterion for the superiority of optimization algorithms over each other is to provide the best quasi-optimal solutions. This has been the main reason to design numerous optimization algorithms by scholars. In this section, optimization algorithms are studied from the perspective of design idea. Optimization algorithms can be divided into four groups based on the idea of design: swarm-based, physics-based, game-based, and evolutionary-based optimization algorithms.

Swarm-based optimization algorithms are developed inspired by natural processes, the behavior of living organisms, plants, and animals. Particle Swarm Optimization (PSO) is one of the oldest and most popular optimization algorithms which is designed based on the behavior of birds in search of food [16]. Artificial Bee Colony (ABC) algorithm is an optimization algorithm based on swarm intelligence and intelligent behavior of the bee population that simulates the behavior of a bee colony [17]. Some of the other swarm-based optimization algorithms are: Seagull Optimization Algorithm (SOA) [18], Group Optimization (GO) [19], Teaching-Learning-Based Optimization (TLBO) [20], Doctor and Patient Optimizer (DPO) [8] Grey Wolf Optimizer (GWO) [21], Following Optimization Algorithm (FOA) [22], Donkey Theorem Optimization (DTO) [23], Rat Swarm Optimizer (RSO) [24], Multi Leader Optimizer (MLO) [25], Emperor Penguin Optimizer (EPO) [26], and "The Good, the Bad, and the Ugly" Optimizer (GBUO) [27].

Physics-based optimization algorithms are developed based on the inspiration of various laws in physics. Gravitational Search algorithm (GSA) is 
developed inspired by the law of gravity between objects at different distances [28]. Momentum Search Algorithm (MSA) is introduced from the simulation of the laws of momentum and motion in a system consisting of a number of spheres [29]. Simulated Annealing (SA) is introduced based on simulation of gradual melting and cooling of metals[30].

Evolutionary-based optimization algorithms are developed based on simulation of various phenomenon in genetic sciences. Genetic algorithm, which is one of the oldest and most widely used optimization algorithms, belongs to this group of optimization algorithms. GA is inspired by the process of reproduction and its simulation in three main stages: parental selection, crossover, and mutation [31]. Artificial Immune System (AIS) is designed based on modeling the performance of the human immune system against diseases and virus attacks [32].

Game-based optimization algorithms have been developed based on ideation on games rules. Football Game-Based Optimization (FGBO) is developed based on simulating the behavior of players and clubs in the football league [33]. Orientation Search Algorithm (OSA) is inspired by the behavior and movement of the players in the direction determined by the referee and the simulation of the rules in the orientation game [34, 35]. Some of the other gamebased optimization algorithms are: Hide Objects Game Optimization (HOGO) [36], Dice Game Optimizer (DGO) [37], Ring Toss Game-Based Optimization (RTGBO) [38], and Shell Game Optimization (SGO) [39].

\section{Mixed Best Members Based Optimizer}

In this section, the theory of the proposed Mixed Best Members Based Optimizer (MBMBO) is described and then mathematically modeled for implementation on solving optimization problems. MBMBO is a population-based algorithm that is able to provide a suitable quasi-optimal solution to an optimization problem during an iterative process. The main idea in designing the MBMBO is to create a mixed member of several top members of the population in order to guide and update the algorithm population.

The population of the algorithm using a matrix called the population matrix is specified in Eq. (1).

$$
X=\left[\begin{array}{c}
X_{1} \\
\vdots \\
X_{i} \\
\vdots \\
X_{N}
\end{array}\right]_{N \times m}=\left[\begin{array}{ccccc}
x_{1,1} & \cdots & x_{1, d} & \cdots & x_{1, m} \\
\vdots & \ddots & \vdots & \ddots & \vdots \\
x_{i, 1} & \cdots & x_{i, d} & \cdots & x_{i, m} \\
\vdots & \ddots & \vdots & \ddots & \vdots \\
x_{N, 1} & \cdots & x_{N, d} & \cdots & x_{N, m}
\end{array}\right]_{N \times m}
$$

Here, $X$ is the population matrix of MBMBO, $X_{i}$ is the $i$ 'th population member, $x_{i, d}$ is the value of $d^{\prime}$ th problem variable specified by $i$ 'th population member, $N$ is the number of population members, and $m$ is the number of problem variables.

Each member of the population matrix is a vector that proposes values for the problem variables. Therefore, based on the values proposed by each member, a value is obtained for the objective function. As a result, the objective function is defined as a vector using Eq. (2).

$$
F=\left[\begin{array}{c}
F_{1} \\
\vdots \\
F_{i} \\
\vdots \\
F_{N}
\end{array}\right]_{N \times 1}=\left[\begin{array}{c}
F_{1}\left(X_{1}\right) \\
\vdots \\
F_{i}\left(X_{i}\right) \\
\vdots \\
F_{N}\left(X_{N}\right)
\end{array}\right]_{N \times 1}
$$

Here, $F$ is the vector of objective function and $F_{i}$ is the value of objective function based on $i$ th population member.

At this stage of modeling, a certain number of top members of the population matrix are selected based on the values of the objective function using Eq. (3) and Eq. (4).

$$
\begin{gathered}
T M=\left[\begin{array}{c}
T M_{1} \\
\vdots \\
T M_{j} \\
\vdots \\
T M_{N}
\end{array}\right]_{N_{T M} \times m} \\
N_{T M}=1+\operatorname{round}\left(0.2 \times N \times \frac{1-t}{T}\right)
\end{gathered}
$$

Here, $T M$ is the matrix of top members, $T M_{i}$ is the $j$ 'th top member, $N_{T M}$ is the number of top members, $t$ is the iteration counter, and $T$ is the maximum of iterations.

After selecting the top members of the population matrix, the mixed member is created based on the averaging of the selected top members using Eq. (5).

$$
M B M=\frac{1}{N_{T M}} \sum_{j=1}^{N_{T M}} T M_{j}
$$

Here, $M B M$ is the mixed best member.

The population matrix update in the proposed MBMBO is modeled based on the mixed best member using Eqs. (6) to (8).

$$
\begin{aligned}
d x_{i, d}= & \left\{\begin{array}{l}
r\left(M B M-x_{i, d}\right), F_{M B M}<F_{i} \\
r\left(x_{i, d}-M B M\right), \text { else }
\end{array}\right. \\
& x_{i, d}^{\text {new }}=x_{i, d}+d x_{i, d}
\end{aligned}
$$




$$
X_{i}=\left\{\begin{array}{cl}
X_{i}^{\text {new }}, & F_{i}^{\text {new }}<F_{i} \\
X_{i}, & \text { else }
\end{array}\right.
$$

Here, $d x_{i, d}$ is the displacement value of $i$ th population member in $d$ 'th dimension, $r$ is a random number in [0 1] interval, $F_{M B M}$ is the objective function value of mixed-best member, $x_{i, d}^{\text {new }}$ is the new suggested position for $i$ 'th population member in $d$ 'th dimension, and $F_{i}^{\text {new }}$ is the objective function value for new suggested position of $i$ th population member.

This process of updating the population members is repeated based on Eqs. (3) to (9) until the algorithm is stopped. After completing the iterations of the algorithm, MBMBO provides the best obtained quasi-optimal solution for the optimization problem. The implementation steps of the proposed MBMBO are shown as a flowchart in Fig. 1.

\section{Simulation study and discussion}

In this section, simulation studies on the performance and power of the proposed MBMBO in solving optimization problems are presented. For this purpose, a set of twenty-three standard objective functions of unimodal, high-dimensional multimodel, and fixed-dimensional multi-model types have been selected to analyze various aspects of the proposed MBMBO. In order to evaluate the optimization results obtained from the MBMBO, these results are compared with the performance of eight other optimization algorithms including (i) popular algorithms: Genetic Algorithm (GA) [31], Particle Swarm Optimization (PSO) [16], (ii) famous algorithms: Gravitational Search Algorithm (GSA) [28], Teaching Learning-Based Optimization (TLBO) [20], Gray Wolf Optimizer (GWO) [21], (iii) latest algorithms: Emperor Penguin Optimizer (EPO) [26], Hide Objects Game Optimization (HOGO) [36], and Shell Game Optimization (SGO) [39]. The two indicators of average (Ave) and standard deviation (std) of quasi-optimal solutions have been used as criteria for comparing the performance of optimization algorithms.

The seven objective functions, including F1 to F7, are unimodal functions. The optimization results of these objective functions using the MBMBO and eight other optimization algorithms are presented in Table 1. The MBMBO has been able to provide the global optimal solution for F1 and F6 objective functions. in addition, MBMBO is the best optimizer for the F2, F3, F4, and F7 objective functions compared to the other eight optimization algorithms. Analysis of the results of this table shows that the proposed MBMBO has a high ability to solve this

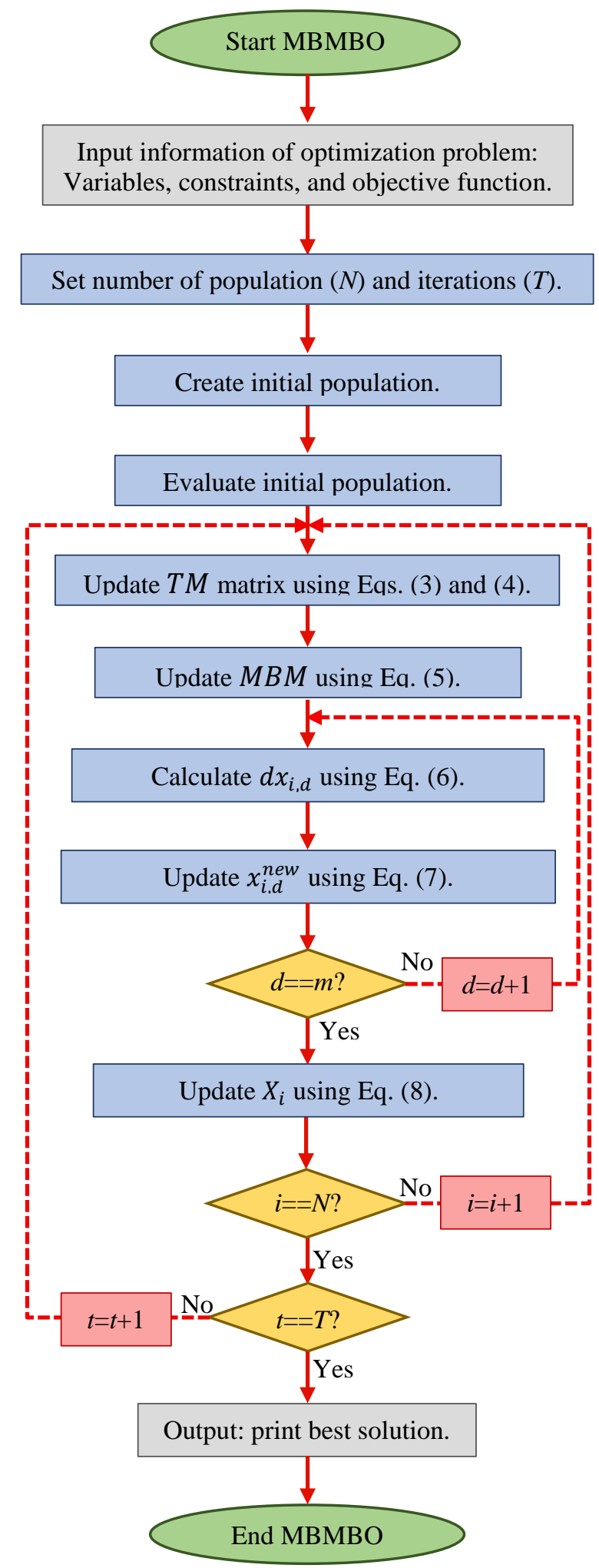

Figure. 1 Flowchart of MBMBO

type of function and is more competitive than other algorithms.

The six objective functions F8 to F13 are selected from the high-dimensional multi-model type. The performance of the MBMBO and eight other optimization algorithms in solving this type of objective functions are presented in Table 2. The 
proposed MBMBO has been able to provide the global optimal solution for the F9, F11, and F13 objective functions by accurately scanning the problem-solving space. The MBMBO is also the best optimizer for the F8, F10, and F12 objective functions than to the eight compared optimization algorithms. Evaluation of the results indicates the optimal performance of the proposed MBMBO in providing quasi-optimal solutions.

The ten objective functions including F14 to F23 are considered to evaluate the performance of the MBMBO in optimization and provide quasi-optimal solutions for fixed-dimensional multi-model objective functions type. The optimization results of these objective functions are presented in Table 3. The MBMBO has performed better in optimizing the F14 and F15 objective functions. In the case of F16, F17, F18, F19, F20, F21, F22, and F23 objective functions, the MBMBO with less standard deviation has been able to provide the global optimal solution more effectively. Analysis of the results of this table shows that the MBMBO is able to solve this type of optimization problems more effectively than similar optimization algorithms.

A general comparison of the optimization results obtained using the proposed $\mathrm{MBMBO}$ and eight other optimization algorithms, shows that the MBMBO has an acceptable ability to solve various optimization problems and is superior and far more competitive than other optimization algorithms.

\subsection{Discussion and theoretical explanation}

Exploitation and exploration capabilities are two special indicators in order to analyze the performance of optimization algorithms.

The exploitation power of an optimization algorithm means the ability of that optimization algorithm to provide a suitable quasi-optimal solution to an optimization problem after the iterations of that algorithm have ended. In fact, according to this concept, optimization algorithms should be able to provide a suitable quasi-optimal solution after full implementation on an optimization problem. Therefore, in comparison the performance of several optimization algorithms, the algorithm that provides the most appropriate quasi-optimal solution and near to optimal global solution has higher exploitation power. The unimodal objective functions, including F1 to F7, have only one main optimal solution and are therefore suitable for evaluating the exploitation power of optimization algorithms. The simulation results of these objective functions using the MBMBO and the other eight optimization algorithms presented in Table 1 show the complete superiority of the proposed MBMBO algorithm over the other algorithms.

Table 1. Results of MBMBO and other algorithms for Unimodal test functions

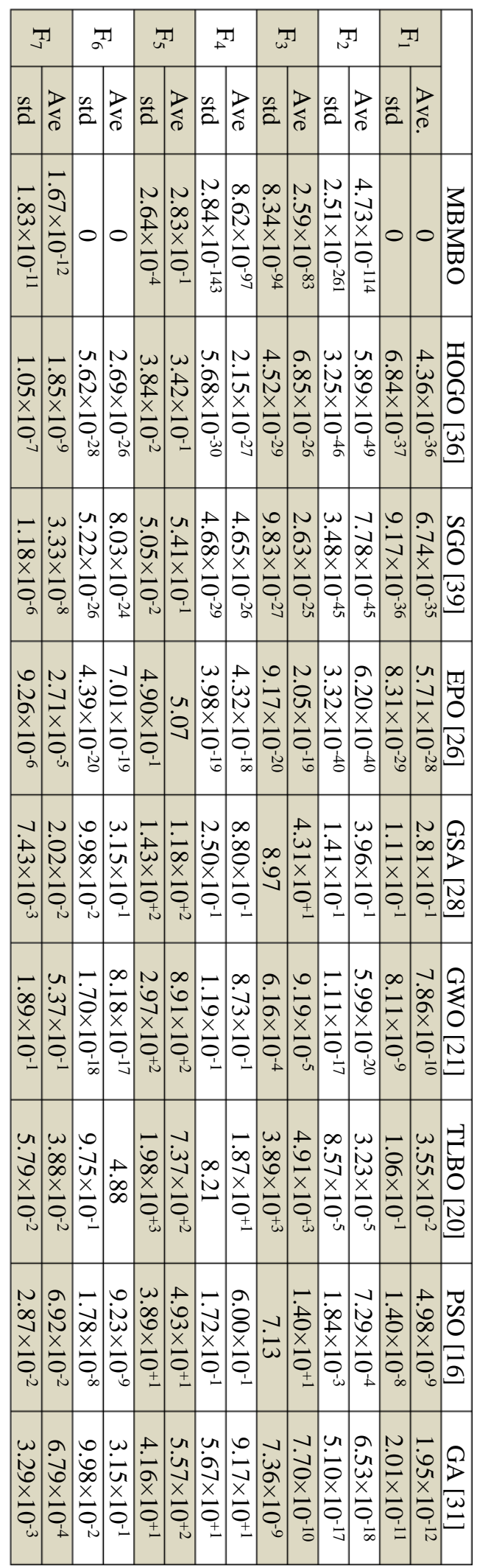


Table 2. Results of MBMBO and other algorithms for high-dimensional Multimodal test functions

\begin{tabular}{|c|c|c|c|c|c|c|c|c|c|c|}
\hline$\frac{T}{\omega}$ & & $\frac{T}{N}$ & & $=$ & $\begin{array}{l}T \\
0\end{array}$ & & T] & & Th & \\
\hline$\frac{n}{2}$ & 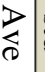 & $\stackrel{2}{2} \frac{d}{d}$ & $\frac{n}{2}$ & $\underset{\infty}{>}$ & $\frac{n}{2}$ & & $\frac{n}{2}$ & 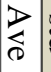 & $\frac{n}{2}$ & 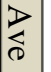 \\
\hline $\begin{array}{l}0 \\
8 \\
8\end{array}$ & $\stackrel{8}{8}$ & 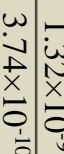 & 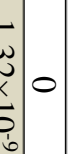 & 0 & 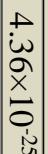 & 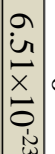 & 0 & 0 & $\begin{array}{c}\dot{u} \\
\vec{x} \\
\dot{x} \\
\vdots \\
\vdots\end{array}$ & $\begin{array}{l}1 \\
\dot{N} \\
x \\
\dot{x} \\
\vdots \\
\pm\end{array}$ \\
\hline
\end{tabular}

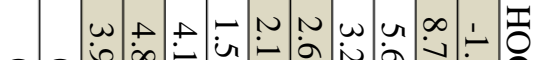

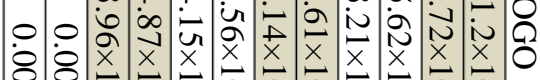
0 O

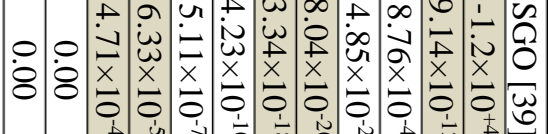
$N-\omega$ u

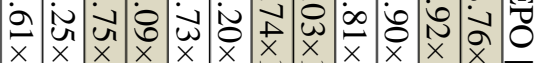
菅

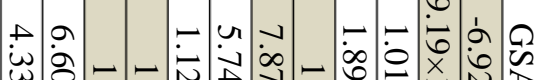
w

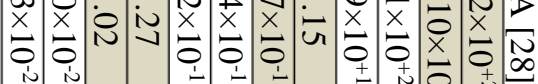

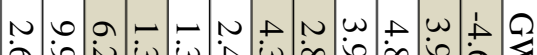

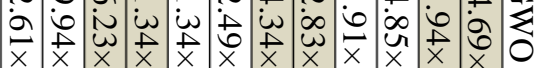
家

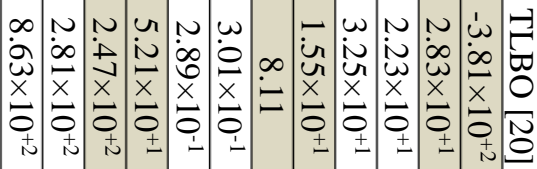

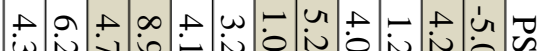
w

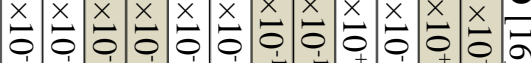

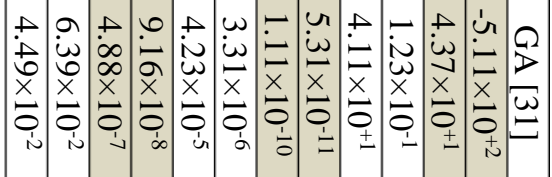

Table 3. Results of MBMBO and other algorithms for fixeddimensional Multimodal test functions

\begin{tabular}{|c|c|c|c|c|c|c|c|c|c|c|c|c|c|c|c|c|c|c|}
\hline 㺼 & & $\mathbb{N}_{N}^{T}$ & & $\mathbb{N}^{\top}$ & & స్రం & 5 & & ${ }_{\infty}^{T}$ & & $\underset{\sim}{T}$ & $\frac{7}{2}$ & $\pi$ & $\frac{T}{u}$ & & $\underset{F}{T}$ & & \\
\hline$\tilde{c}$ & 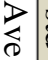 & $\frac{a}{2}$ & 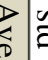 & 2 & 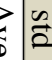 & 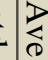 & $\underline{\tilde{a}}$ & 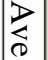 & $\underline{a}$ & 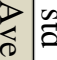 & 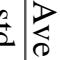 & $\stackrel{\tilde{a}}{a}$ & 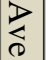 & $\underline{a}$ & 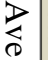 & $\stackrel{a}{a}$ & 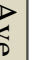 & \\
\hline 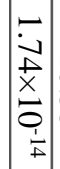 & $\begin{array}{l}1 \\
0 \\
\dot{u} \\
u \\
u\end{array}$ & 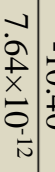 & 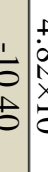 & 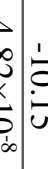 & 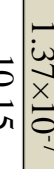 & 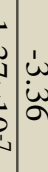 & 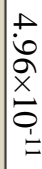 & $\begin{array}{l}\omega \\
\dot{\alpha} \\
\alpha \\
\alpha\end{array}$ & 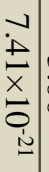 & $\omega$ & 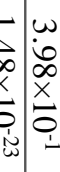 & 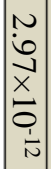 & $\stackrel{.}{\dot{\omega}}$ & $\begin{array}{l}\omega \\
\underset{\infty}{\infty} \\
\dot{x} \\
\overrightarrow{0} \\
\dot{\sim}\end{array}$ & 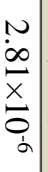 & 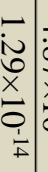 & $\begin{array}{l}+ \\
\infty \\
\dot{1} \\
x \\
\vec{\sigma} \\
\dot{\alpha}\end{array}$ & 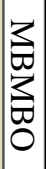 \\
\hline
\end{tabular}

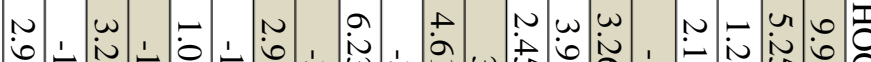

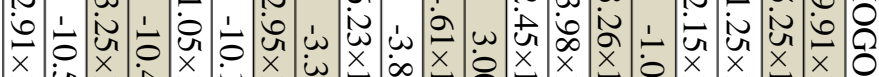

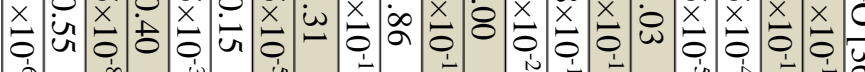

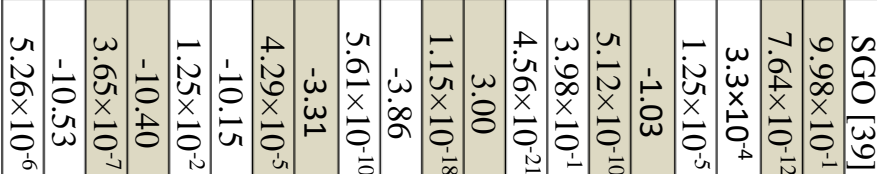

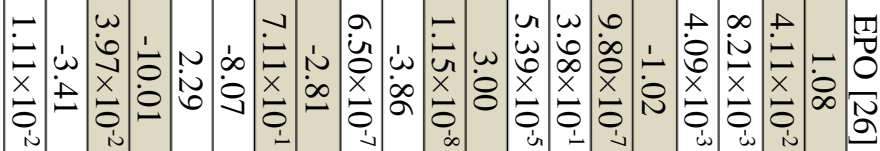

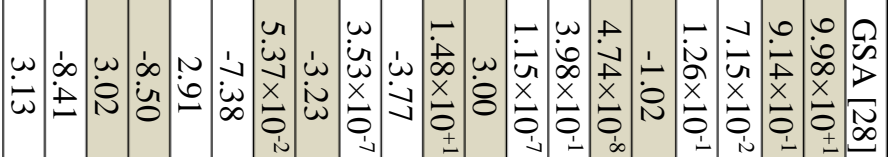

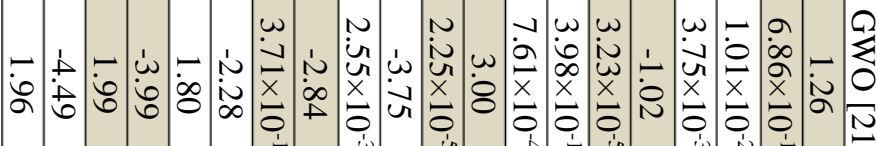

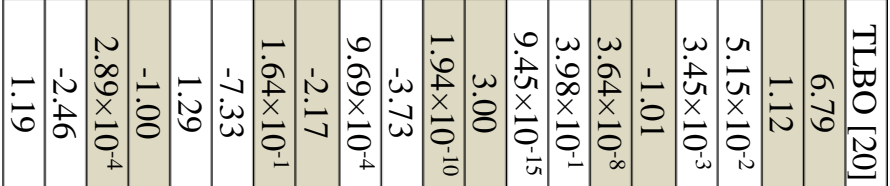

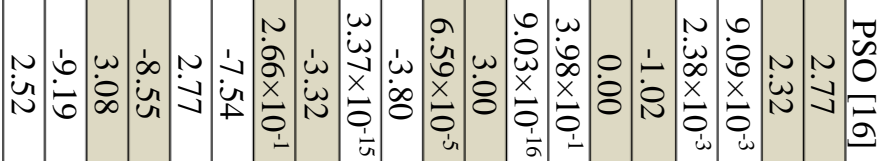

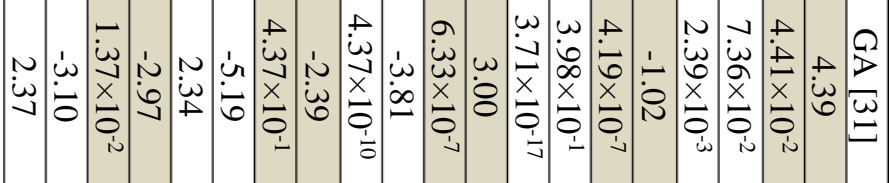

Therefore, the MBMBO has high exploitation power in solving optimization problems and providing a suitable quasi-optimal solution.

The exploration power of an optimization 
algorithm means the ability of that optimization algorithm to accurately search the problem-solving space. Therefore, in analyzing the performance of several optimization algorithms, an algorithm that can search the problem-solving space more accurately has better exploration power. This indicator is especially important when solving complex optimization problems that have local optimal solutions. High dimensional multi-modal objective functions including F8 to F13 and also fixed dimensional multi-modal objective functions including F14 to F23 have several local solutions. Therefore, these objective functions are suitable for analyzing the exploration index of optimization algorithms. The optimization results of these objective functions, presented in Table 2 and Table 3 , show the acceptable exploration ability of the MBMBO in the exact search of the problem-solving space.

\section{Conclusion and future works}

In this paper a new population-based optimization algorithm called Mixed Best Members Based Optimizer (MBMBO) has been introduced that can be used to solve various optimization problems. The main idea in designing the proposed MBMBO was to create a mixed member of several top members of the population in order to guide and update the algorithm population. The proposed MBMBO was mathematically modelled to be implemented on optimization problems. The performance of the MBMBO in providing suitable quasi-optimal solutions was evaluated on a set of twenty-three standard objective functions of types unimodal, high-dimensional multi-model, and fixeddimensional multi-model. Unimodal objective functions that have only one main optimal solution has been considered to analyse the exploitation index. The optimization results of these objective functions indicated the high exploitation power of the MBMBO in solving optimization problems. High-dimensional multi-model and fixed-dimensional multi-model objective functions that have several local solutions in addition to the main optimal have been considered to evaluate the exploration index. The optimization results of these types of objective functions indicated the high power of the proposed MBMBO in the exploration index. optimization results obtained from the MBMBO, have been compared with the performance of eight other optimization algorithms including Genetic Algorithm (GA), Particle Swarm Optimization (PSO), Gravitational Search Algorithm (GSA), Teaching Learning-Based Optimization (TLBO), Gray Wolf Optimizer (GWO), Emperor
Penguin Optimizer (EPO), Hide Objects Game Optimization (HOGO), and Shell Game Optimization (SGO).

The optimization results showed the acceptable ability of the proposed algorithm to solve various optimization problems. In addition, a comparison of the simulation results shows that the MBMBO is superior to the other eight optimization algorithms and is much more competitive.

The authors suggest some ideas and perspectives for future studies. Design of the binary version as well as multi-objective version of MBMBO is an interesting topic for future investigations. Moreover, implementing $\mathrm{MBMBO}$ on various optimization problems and real-world optimization problems could achieve some significant contributions, as well.

\section{Conflicts of Interest}

The authors declare no conflict of interest.

\section{Author Contributions}

Conceptualization, S.A.D and F.A.Z.; methodology, M.D.; software, S.A.D. and M.D.; validation, F.A.Z., O.P.M., and M.D.; formal analysis, O.P.M. and F.A.Z.; investigation, M.D., S.A.D., and O.P.M.; resources, M.D.; data curation, F.A.Z.; writing - original draft preparation, S.A.D.; writing - review and editing, O.P.M. and F.A.Z; visualization, M.D; supervision, M.D.; project administration, S.A.D and F.A.Z.; funding acquisition, O.P.M.

\section{References}

[1] M. Dorigo and T. Stützle, "Ant colony optimization: overview and recent advances", Handbook of metaheuristics, pp. 311-351, 2019.

[2] M. Dehghani, Z. Montazeri, A. Dehghani, and A. Seifi, "Spring Search Algorithm: A New MetaHeuristic Optimization Algorithm Inspired by Hooke's Law", In: Proc. of IEEE 4th International Conference on Knowledge-Based Engineering and Innovation (KBEI), Tehran, Iran, pp. 210-214, 2017.

[3] M. Dehghani, Z. Montazeri, H. Givi, J. M. Guerrero, and G. Dhiman, "Darts game optimizer: A new optimization technique based on darts game", International Journal of Intelligent Engineering and Systems, Vol. 13, pp. 286-294, 2020.

[4] M. Dehghani, Z. Montazeri, and O. P. Malik, "ENERGY COMMITMENT: A PLANNING OF ENERGY CARRIER BASED ON ENERGY 
CONSUMPTION", Electrical Engineering \& Electromechanics, No. 4, pp. 69-72, 2019.

[5] M. Dehghani, M. Mardaneh, O. P. Malik, J. M. Guerrero, C. Sotelo, D. Sotelo, M. Nazari-Heris, K. Al-Haddad, and R. A. Ramirez-Mendoza, "Genetic Algorithm for Energy Commitment in a Power System Supplied by Multiple Energy Carriers", Sustainability, Vol. 12, No. 23, pp. 10053, 2020.

[6] A. Ehsanifar, M. Dehghani, and M. Allahbakhshi, "Calculating The Leakage Inductance for Transformer Inter-Turn Fault Detection Using Finite Element Method", In: Proc. of Iranian Conference on Electrical Engineering (ICEE), pp. 1372-1377, 2017.

[7] M. Dehghani, M. Mardaneh, O. P. Malik, J. M. Guerrero, R. Morales-Menendez, R. A. RamirezMendoza, J. Matas, and A. Abusorrah, "Energy Commitment for a Power System Supplied by Multiple Energy Carriers System using Following Optimization Algorithm", Applied Sciences, Vol. 10, No. 17, pp. 5862, 2020.

[8] M. Dehghani, M. Mardaneh, J. M. Guerrero, O. P. Malik, R. A. Ramirez-Mendoza, J. Matas, J. C. Vasquez, and L. Parra-Arroyo, "A new "Doctor and Patient" optimization algorithm: An application to energy commitment problem", Applied Sciences, Vol. 10, No. 17, pp. 5791, 2020.

[9] M. Dehghani, Z. Montazeri, and O. Malik, "Optimal sizing and placement of capacitor banks and distributed generation in distribution systems using spring search algorithm", International Journal of Emerging Electric Power Systems, Vol. 21, No. 1, 2020.

[10] M. Dehghani, Z. Montazeri, O. P. Malik, K. AlHaddad, J. M. Guerrero, and G. Dhiman, "A New Methodology Called Dice Game Optimizer for Capacitor Placement in Distribution Systems", Electrical Engineering \& Electromechanics, No. 1, pp. 61-64, 2020.

[11] M. Dehghani, M. Mardaneh, Z. Montazeri, A. Ehsanifar, M. J. Ebadi, and O. M. Grechko, "Spring search algorithm for simultaneous placement of distributed generation and capacitors", Electrical Engineering \& Electromechanics, No. 6, pp. 68-73, 2018.

[12] S. Dehbozorgi, A. Ehsanifar, Z. Montazeri, M. Dehghani, and A. Seifi, "Line Loss Reduction and Voltage Profile Improvement in Radial Distribution Networks Using Battery Energy Storage System", In: Proc. of IEEE 4th International Conference on Knowledge-Based Engineering and Innovation (KBEI), pp. 02150219, 2017.
[13]Z. Montazeri and T. Niknam, "Optimal utilization of electrical energy from power plants based on final energy consumption using gravitational search algorithm", Electrical Engineering \& Electromechanics, No. 4, pp. 7073, 2018.

[14] M. Dehghani, Z. Montazeri, A. Ehsanifar, A. R. Seifi, M. J. Ebadi, and O. M. Grechko, "Planning of energy carriers based on final energy consumption using dynamic programming and particle swarm optimization", Electrical Engineering \& Electromechanics, No. 5, pp. 6271, 2018.

[15] Z. Montazeri and T. Niknam, "Energy Carriers Management Based on Energy Consumption", In: Proc. of IEEE 4th International Conference on Knowledge-Based Engineering and Innovation (KBEI), pp. 0539-0543, 2017.

[16] J. Kennedy and R. Eberhart, "Particle swarm optimization", in Proceedings of ICNN'95international conference on neural networks, Vol. 4, ed: IEEE, pp. 1942-1948, 1995.

[17] D. Karaboga and B. Basturk, "Artificial bee colony (ABC) optimization algorithm for solving constrained optimization", International fuzzy systems association world congress, ed: Springer, pp. 789-798, 2007.

[18] G. Dhiman, K. K. Singh, M. Soni, A. Nagar, M. Dehghani, A. Slowik, A. Kaur, A. Sharma, E. H. Houssein, and K. Cengiz, "MOSOA: A new multi-objective seagull optimization algorithm", Expert Systems with Applications, pp. 114150, 2020.

[19] M. Dehghani, Z. Montazer, A. Dehghani, and O. P. Malik, "GO: Group Optimization", Gazi University Journal of Science, Vol. 33, 2020.

[20]R. V. Rao, V. J. Savsani, and D. Vakharia, "Teaching-learning-based optimization: a novel method for constrained mechanical design optimization problems", Computer-Aided Design, Vol. 43, No. 3, pp. 303-315, 2011.

[21] S. Mirjalili, S. M. Mirjalili, and A. Lewis, "Grey wolf optimizer", Advances in engineering software, Vol. 69, pp. 46-61, 2014.

[22] M. Dehghani, M. Mardaneh, and O. Malik, "FOA:'Following'Optimization Algorithm for solving Power engineering optimization problems", Journal of Operation and Automation in Power Engineering, Vol. 8, No. 1, pp. 57-64, 2020.

[23] M. Dehghani, M. Mardaneh, O. P. Malik, and S. M. NouraeiPour, "DTO: Donkey Theorem Optimization", In: Proc. of 27th Iranian Conference on Electrical Engineering (ICEE), pp. 1855-1859, 2019. 
[24] G. Dhiman, M. Garg, A. K. Nagar, V. Kumar, and M. Dehghani, "A Novel Algorithm for Global Optimization: Rat Swarm Optimizer", Journal of Ambient Intelligence and Humanized Computing, 2020.

[25] M. Dehghani, Z. Montazeri, A. Dehghani, R. R. Mendoza, H. Samet, J. M. Guerrero, and G. Dhiman, "MLO: Multi Leader Optimizer", International Journal of Intelligent Engineering and Systems, Vol. 13, No. 6, pp. 364-373, 2020.

[26] G. Dhiman and V. Kumar, "Emperor Penguin Optimizer: A Bio-inspired Algorithm for Engineering Problems", Knowledge-Based Systems, 2018.

[27] H. Givi, M. Dehghani, Z. Montazeri, R. Morales-Menendez, R. A. Ramirez-Mendoza, and N. Nouri, "GBUO: "The Good, the Bad, and the Ugly" Optimizer", Applied Sciences, Vol. 11, No. 5, pp. 2042, 2021.

[28] E. Rashedi, H. Nezamabadi-Pour, and S. Saryazdi, "GSA: a gravitational search algorithm", Information Sciences, Vol. 179, No. 13, pp. 2232-2248, 2009.

[29] M. Dehghani and H. Samet, "Momentum search algorithm: A new meta-heuristic optimization algorithm inspired by momentum conservation law", SN Applied Sciences, Vol. 2, No. 10, pp. 115, 2020.

[30] P. J. Van Laarhoven and E. H. Aarts, "Simulated annealing", Simulated annealing: Theory and applications, ed: Springer, pp. 7-15, 1987.

[31] A. Bose, T. Biswas, and P. Kuila, "A novel genetic algorithm based scheduling for multicore systems", Smart Innovations in Communication and Computational Sciences, ed: Springer, pp. 45-54, 2019.

[32] S. A. Hofmeyr and S. Forrest, "Architecture for an artificial immune system", Evolutionary computation, Vol. 8, No. 4, pp. 443-473, 2000.

[33] M. Dehghani, M. Mardaneh, J. M. Guerrero, O. Malik, and V. Kumar, "Football game based optimization: An application to solve energy commitment problem", International Journal of Intelligent Engineering and Systems, Vol. 13, pp. 514-523, 2020.

[34] M. Dehghani, Z. Montazeri, O. P. Malik, A. Ehsanifar, and A. Dehghani, "OSA: Orientation search algorithm", International Journal of Industrial Electronics, Control and Optimization, Vol. 2, No. 2, pp. 99-112, 2019.

[35] M. Dehghani, Z. Montazeri, O. P. Malik, G. Dhiman, and V. Kumar, "BOSA: Binary Orientation Search Algorithm", International Journal of Innovative Technology and Exploring
Engineering (IJITEE), Vol. 9, No. 1, pp. 53065310, 2019.

[36] M. Dehghani, Z. Montazeri, S. Saremi, A. Dehghani, O. P. Malik, K. Al-Haddad, and J. M. Guerrero, "HOGO: Hide Objects Game Optimization", International Journal of Intelligent Engineering and Systems, Vol. 13, No. 4, pp. 216-225, 2020.

[37] M. Dehghani, Z. Montazeri, and O. P. Malik, "DGO: Dice game optimizer", Gazi University Journal of Science, Vol. 32, No. 3, pp. 871-882, 2019.

[38] S. A. Doumari, H. Givi, M. Dehghani, and O. P. Malik, "Ring Toss Game-Based Optimization Algorithm for Solving Various Optimization Problems", International Journal of Intelligent Engineering and Systems, Vol. 14, No. 3, pp. 545-554, 2021.

[39] D. Mohammad, M. Zeinab, O. P. Malik, H. Givi, and J. M. Guerrero, "Shell Game Optimization: A Novel Game-Based Algorithm", International Journal of Intelligent Engineering and Systems, Vol. 13, No. 3, pp. 246-255, 2020. 